\title{
SOME REMARKS ON HYPERSPACES
}

\author{
RAYMOND Y. T. WONG ${ }^{1}$
}

1. The purpose of this paper is to answer a question of R. Schori [3] and to provide simpler arguments for some generalizations of Schori's results.

If $X$ is a metric space, the hyperspace of $X$, denoted $2^{X}$, is the space of all nonvoid closed subsets of $X$ with the usual Hausdorff metric. The $n$-fold $(n \geqq 1)$ symmetric product (Borsuk-Ulam [1]) of $X$, denoted $X(n)$, is the subspace of $2^{X}$ consisting of all elements with $\leqq n$ points. Let $I$ denote the closed unit interval, $I^{n}$ the $n$-cube and $I^{\infty}$ the Hilbert cube. Let $S(X)$ denote the subspace of $2^{x}$ consisting of all continua. In [3] R. Schori shows that for $n \geqq 1$ and $\alpha=\infty, 1,2$, $\cdots, I^{\alpha}(n)$ contains $I^{\alpha}$ as a factor; that is, $I^{\alpha}(n)$ is homeomorphic to $Y \times I^{\alpha}$ for some space $Y$. Let $J^{\infty}$ denote another copy of the Hilbert cube with $J=[-1,1]$ and let $R$ be the equivalence relation on $J^{\infty}$ defined by identifying each $x=\left(x_{1}, x_{2}, \cdots\right)$ with $-x$ $=\left(-x_{1},-x_{2}, \cdots\right)$.

THEOREM I. $J^{\infty} / R$ is not homeomorphic to $J^{\infty}$.

Thus we settle a question of R. Schori [3].

Proof. Let us suppose it were. Consider the natural quotient map $P: J^{\infty} \rightarrow J^{\infty} / R$. Evidently the restriction of $P: J^{\infty}-0 \rightarrow J^{\infty} / R-P(0)$ is a two-fold covering. Since the Hilbert cube is homogeneous, it follows from the assumption that $J^{\infty} / R-P(0)$ is simply connected and therefore (well-known) does not admit a two-fold covering. This is a contradiction.

Question. Is $J^{\infty} / R$ an Absolute Retract?

The question is interesting because $J^{\infty} / R$ is clearly a retract of $J^{\infty} / R \times J^{\infty}$, which is homeomorphic to $J^{\infty}(2)$ by [3]. A negative answer would imply that $J^{\infty}(2)$ is not homeomorphic to $J^{\infty}$.

Theorem II. Let $m, n$ be positive integers. If $X=I^{m}(n), 2^{\left(I^{m}\right)}$ or $S\left(I^{m}\right)$, then $X$ contains $I^{m}$ as a factor.

REMARK. Schori's proof is restricted to symmetric products since it makes strong use of the following well-known characterization of $I^{m}(n)$. If $n$ is a positive integer, then $I^{m}(n)$ is homeomorphic to $I^{m} / R$ where $R$ is the equivalent relation on $I^{m}$ defined by $\left(x_{1}, \cdots, x_{n}\right)$

Received by the editors June 17, 1968.

${ }^{1}$ Research supported in part by the National Research Council and the Office of Naval Research Grant ONR:432:LDB:lcd. 
$R\left(y_{1}, \cdots, y_{n}\right)$ iff $\left\{x_{1}, \cdots, x_{n}\right\}=\left\{y_{1}, \cdots, y_{n}\right\}\left(x_{i}\right.$ and $y_{i}$ are points in $\left.I^{m}\right)$. However, as such he is able to include the case when $m=\infty$ in his theorem. On the other hand, by working directly with the subspaces of $2^{I^{m}}$ we are able to give a much simplified proof and although we are not able to include $m=\infty$, we generalize in the direction of more general subspaces of $2^{I^{m}}$ which, as the nature of the technique, may include even more subclasses than those mentioned in Theorem II. In the case when $m=\infty$ we are able to prove the following partial generalization:

THEOREM III. If $X=I^{\infty}(n), S\left(I^{\infty}\right)$ or $2^{I^{\infty}}$, then for any positive integer $k, X$ contains $I^{k}$ as a factor.

Question. If $X=S\left(I^{\infty}\right)$ or $2^{I^{\infty}}$, must $X$ contain $I^{\infty}$ as a factor?

2. The Cone Lemma. The cone over a space $X$, denoted $C(X)$, is the quotient space of $X \times I$ obtained by identifying $X \times 1$ as a point $v$, where $v$ is called the vertex of $C(X)$. Inductively for $n>1$, define $C^{n}(X)=C\left(C^{n-1}(X)\right)$. Let " $\approx$ " denote "homeomorphic to".

Lemma. (Schori). For $n>1, C^{n}(X) \approx C(X) \times I^{n-1}$.

Outline of ProOF. By induction it suffices to consider $n=2$; that is $C^{2}(X) \approx C(X) \times I$. For each $x \in X, C^{2}(x)$ can be realized as a triangle and thus we can deform $C^{2}(x)$ into $C(x) \times I$. If we do this uniformly for each $x$ (detail in [3]), we obtain a homeomorphism from $C^{2}(X)$ onto $C(X) \times I$.

Proof of Theorem II. Let $\left\{v_{i}\right\}$ be the unit points in Euclidean space $E^{m+1}$; that is, $v_{i}$ has 1 for its $i$ th-coordinate and 0 otherwise. Let $\sigma$ denote the $m$-simplex $v_{1} v_{2} \cdots v_{m+1}$. Since $\sigma \approx I^{m}$, it is clear we can replace $I^{m}$ by $\sigma$ in Theorem II. For each $i$ let $\sigma_{i}$ be the $(m-1)$ dimensional face $v_{1} \cdots \hat{v}_{i} \cdots v_{m+1}$. Now let $X_{0}$ be any space in Theorem II. For $i \geqq 1$ let $X_{i}=\left\{x \in X_{0} \mid x \cap \sigma_{k} \neq \varnothing\right.$ for all $\left.k \leqq i\right\}$. Clearly $C\left(X_{i+1}\right) \approx\left\{t v_{i+1}+(1-t) x: t \in I, x \in X_{i+1}\right\}_{1} \subset X_{i}$. We contend that $\left\{t v_{i+1}+(1-t) x: t \in I, x \in X_{i+1}\right\}=X_{i}$. Suppose $x\left(\neq v_{i+1}\right) \in X_{i}$. Let $t=\min \pi_{i+1}(x)$, where $\pi_{i+1}$ is the usual projection map. It is routine to verify that $x^{\prime}=x /(1-t)-t v_{i+1} / \in X_{i+1}$ and thus $t v_{i+1}+(1-t) x^{\prime}$ $=x$. Inductively, we have $X_{0} \approx C\left(X_{1}\right) \approx C C\left(X_{2}\right) \cdots \approx C^{m+1}\left(X_{m+1}\right)$. The theorem now follows from the Cone Lemma.

Proof of Theorem III. Let $s$ denote the infinite product of reals and let $T=\left\{\left(x_{1}, x_{2}, \cdots\right) \in I^{\infty} \mid 0 \leqq x_{i} \leqq 1\right.$ and $\left.\sum_{i=1}^{\infty} x_{i} \leqq 1\right\}$. Evidently $T$ is closed in $I^{\infty}$ and therefore compact. Thus $T$ is a compact convex subset of the locally convex topological linear space $s$ which admits a countable family of continuous linear forms that separate 
points (namely, the family $\left\{\pi_{i}\right\}$ of projections) and thus by [2] $T$ is homeomorphic to $I^{\infty}$. Hence we may replace $I^{\infty}$ by $T$ in Theorem III. Now let $X_{0}$ be any space in Theorem III and let $k$ be any positive integer. For each $i \geqq 1$, let $T_{i}=\left\{\left(x_{1}, x_{2}, \cdots\right) \in T \mid x_{i}=0\right\}$ and $X_{i}$ $=\left\{x \in X_{0} \mid x \cap T_{k} \neq \varnothing\right.$ for all $\left.k \leqq i\right\}$. As in Theorem II, $C\left(X_{i+1}\right) \approx X_{i}$. Inductively, $X_{0} \approx C\left(X_{1}\right) \cdots \approx C^{k+1}\left(X_{k+1}\right)$. The theorem now follows from the Cone Lemma.

\section{REFERENCES}

1. K. Borsuk and S. Ulam, On symmetric products of topological spaces, Bull. Amer. Math. Soc. 37 (1931), 875-882.

2. V. L. Klee, Jr., Some topological properties of convex sets, Trans. Amer. Math. Soc. 78 (1955), 30-45.

3. R. Schori, Hyperspaces and symmetric products of topological spaces, Fund. Math. 63 (1968), 77-88.

UNIVERSITY OF WASHINGTON 